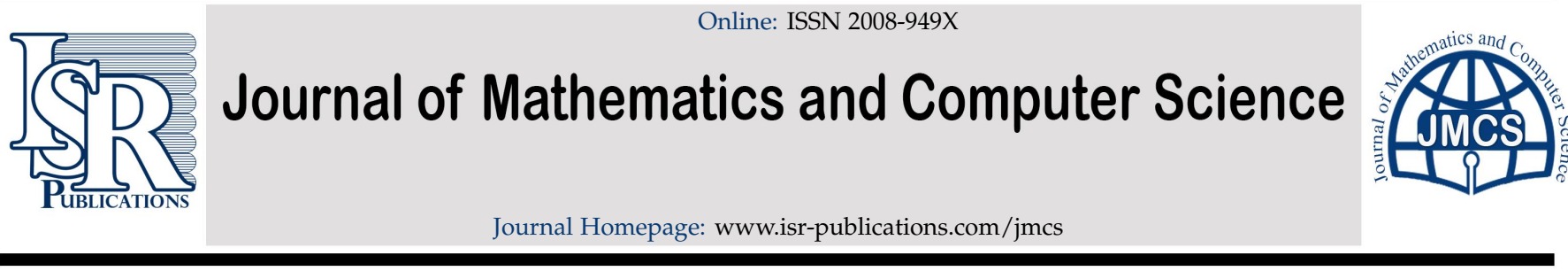

\title{
Dynamics of some parametric operators from the class of $\zeta^{(\text {as })}$-QSO
}

\section{Basma M. Al-Shutnawi}

Department of Mathematics, Faculty of Science, Tafila Technical University, Tafila 66110, Jordan.

\begin{abstract}
In this paper the quadratic stochastic operators (QSO) were considered, these operators describe the population dynamic system. Some quadratic stochastic operators were studied by Lotka and Volterra. Moreover, we discuss the dynamic of some parametric operators from the class of $\zeta^{(\text {as })}$-QSO.
\end{abstract}

Keywords: Quadratic stochastic operators, $\zeta^{(\text {as })}-\mathrm{QSO}$, fixed points.

2010 MSC: 58F08, 37C25.

(C)2019 All rights reserved.

\section{Introduction}

The history of the quadratic stochastic operators refers to Bernshtein (1924). Many papers were published to develop this theory. Recently many researchers interest in connection with its numerous applications in many branches of mathematics, biology [1, 6-10, 16] and physics [14, 15]. Let $x^{(0)}=\left(x_{1}^{(0)}, x_{2}^{(0)}, \ldots, x_{m}^{(0)}\right)$ be the probability distribution of species in the initial generations, and $P_{i j, k}$ the probability that individuals in the $i^{\text {th }}$ and $j^{\text {th }}$ species interbreed to produce an individual $k$. Then the probability distribution $x^{(1)}=\left(x_{1}^{(1)}, x_{2}^{(1)}, \ldots, x_{m}^{(1)}\right)$ of the species in the first generation can be found by the total probability, $x_{k}^{(1)}=\sum_{i, j=1}^{m} P_{i j, k} x_{i}^{(0)} x_{j}^{(0)}, k=1, \ldots, m$. This defines a map $V$ called evolution operator as follow $\mathrm{V}: x^{(0)} \rightarrow x^{(1)}$, i.e., $\mathrm{V}\left(x^{(0)}\right)=x^{(1)}$ the first generation, and $\mathrm{V}\left(x^{(1)}\right)=x^{(2)}$ the second generation and so $V\left(x^{(n-1)}\right)=x^{(n)}$ is the $n^{\text {th }}$ generation. This describes the population dynamical system. In this paper we discuss the dynamic of some parametric operators from the class of $\zeta^{(\text {as })}-\mathrm{QSO}$ that was generated in [13].

\section{Quadratic stochastic operators}

The quadratic stochastic operator (QSO) is a mapping of the simplex

$$
S^{m-1}=\left\{\mathbf{x}=\left(x_{1}, x_{2}, \ldots, x_{m}\right) \in \mathbb{R}^{m}: x_{i} \geqslant 0: \sum_{i=1}^{m} x_{i}=1\right\}
$$

Email address: Basma@ttu.edu.jo (Basma M. Al-Shutnawi)

doi: $10.22436 /$ jmcs.019.03.08

Received: 2019-03-27 Revised: 2019-05-02 Accepted: 2019-05-10 
into itself of the form

$$
V=x_{k}^{\prime}=\sum_{i, j=1}^{m} P_{i j, k} x_{i} x_{j}, k=1,2, \ldots, m,
$$

where $\left\{P_{i j, k}\right\}$ are heredity coefficients that satisfy the following conditions

$$
P_{i j, k} \geqslant 0, \quad \sum_{k=1}^{m} P_{i j, k}=1, \quad i, j, k=1, \ldots, m .
$$

Note that each element $x \in S^{m-1}$ can be considered as a probability distribution on the set $E=\{1,2, \ldots, m\}$. For a given $x^{(0)} \in S^{m-1}$, the trajectory $\left\{x^{(\mathfrak{n})}\right\}_{\mathfrak{n}=0}^{\infty}$ induced by the QSO (2.1) is defined by

$$
x^{(n+1)}=V\left(x^{(n)}\right) \text {, where } n=0,1,2, \ldots
$$

The asymptotic behavior of the trajectories problem was solved for the voltera QSO's (see [3-5]) with the assumption

$$
P_{i j, k}=0 \quad \text { if } k \notin\{i, j\} .
$$

Where the assumption (2.2) biologically means that every individual repeats the genotype of one of its parents for the relevant QSO

$$
\mathrm{V}: x \in \mathrm{S}^{\mathrm{m}-1} \rightarrow \mathrm{V}(\mathrm{x})=\mathrm{x}^{\prime} \in \mathrm{S}^{\mathrm{m}-1} .
$$

The following formula for Volterra-QSO was given in [3]

$$
x_{k}^{\prime}=x_{k}\left(1+\sum_{i=1}^{m} a_{k i} x_{i}\right)
$$

In [2], the following family of QSO's was considered

$$
\mathrm{V}_{\lambda}: S^{2} \rightarrow \mathrm{S}^{2}, \quad \mathrm{~V}_{\lambda}=(1-\lambda) \mathrm{V}_{0}+\lambda \mathrm{V}_{1}, \quad 0 \leqslant \lambda \leqslant 1,
$$

where $V_{0}(x)=\left(x_{1}^{2}+2 x_{1} x_{2}, x_{2}^{2}+2 x_{2} x_{3}, x_{3}^{2}+2 x_{1} x_{3}\right)$ is a Volterra operator. A fixed point of an operator $V$ can be defined as a solution of the equation $V(x)=x$. Let us define $\operatorname{Fix}(V)=\left\{x \in S^{m-1}: V(x)=x\right\}$ as a fixed point set, due to Brouwer's theorem $\operatorname{Fix}(\mathrm{V}) \neq \phi$. In this paper we discuss the dynamic of some parametric operators from the class of $\zeta^{(a s)}$-QSO. Let us denote the set

$$
P_{m}=\{(i, j): i \leqslant j\} \subset I \times I, \quad I=\{1,2, \ldots, m\} \text { and } \triangle_{m}=\{(i, i): i \in I\} \subset I \times I .
$$

And $\mathbb{P}_{i j}=\left\{P_{i j, 1}, \ldots, P_{i j, m}\right\}$ for a given pair $(i, j) \in P_{m} \cup \triangle_{m}$, hence $\mathbb{P}_{i j} \in S^{m-1}$.

Let $\zeta_{1}=\left\{A_{i}\right\}_{i=1}^{N}$ and $\zeta_{2}=\left\{B_{i}\right\}_{i=1}^{M}$ be some fixed partitions of $P_{m}$ and $\triangle_{m}$, respectively, i.e., $A_{i} \cap A_{j}=\phi$, $B_{i} \cap B_{j}=\phi$ and $\cup_{i=1}^{N} A_{i}=P_{m}, \cup_{i=1}^{N} B_{i}=\triangle_{m}$, where $N, M \leqslant m$.

Definition 2.1. An operator $V: S^{m-1} \rightarrow S^{m-1}$, defined by $V=x_{k}^{\prime}=\sum_{i, j=1}^{m} P_{i j, k} x_{i} x_{j}$ for $k=1,2, \ldots, m$, where $P_{i j, k} \geqslant 0, \sum_{k=1}^{m} P_{i j, k}=1$ for $i, j, k=1, \ldots, m$ is called a $\zeta^{(\text {as })}$-QSO w.r.t the partitions $\zeta_{1}, \zeta_{2}$ if the following conditions are satisfied.

(i) $\mathbb{P}_{i j} \sim \mathbb{P}_{u v}$ for any $(i, j),(u, v) \in A_{k}$ and for each $k \in\{1, \ldots, N\}$.

(ii) $\mathbb{P}_{i j} \perp \mathbb{P}_{u v}$ for any $(i, j) \in A_{k}$ and $(u, v) \in A_{l}$, and for any $k \neq l, k, l \in\{1, \ldots, N\}$.

(iii) $\mathbb{P}_{i i} \sim \mathbb{P}_{j j}$ for any $(i, i),(j, j) \in B_{d}$, for each $d \in\{1, \ldots, M\}$.

(iv) $\mathbb{P}_{u u} \perp \mathbb{P}_{v v}$ for any $(u, u) \in B_{s}$ and $(v, v) \in B_{h}$, for any $s \neq h, s, h \in\{1, \ldots, M\}$.

Some classes of $\zeta^{(\text {as })}-$ QSOs have been studied in [11-13], in [13] the authors studied $\zeta^{(s)}-$ QSO in two-dimensional simplex, that is $\mathrm{m}=3$. In this case they have the following possible partitions of $\mathrm{P}_{3}$ 


$$
\begin{aligned}
& \zeta_{1}=\{\{(1,2)\},\{(1,3)\},\{(2,3)\}\}, \quad\left|\zeta_{1}\right|=3, \\
& \zeta_{2}=\{\{(2,3)\},\{(1,2),(1,3)\}\}, \quad\left|\zeta_{2}\right|=2 \\
& \zeta_{3}=\{\{(1,3)\},\{(1,2),(2,3)\}\}, \quad\left|\zeta_{3}\right|=2 \\
& \zeta_{4}=\{\{(1,2)\},\{(1,3),(2,3)\}\}, \quad\left|\zeta_{4}\right|=2 \\
& \zeta_{5}=\{(1,2),(1,3),(2,3)\}, \quad\left|\zeta_{1}\right|=1 .
\end{aligned}
$$

In the same paper they showed that any $\zeta^{(s)}$-QSO taken from the class corresponding to partition $\zeta_{3}$ is conjugated to some $\zeta^{(s)}$-QSO taken from the class corresponding to partition $\zeta_{2}$. Therefore, they saw that it is enough to study a class of all $\zeta^{(s)}-\mathrm{QSO}$ corresponding to the partition $\zeta_{2}$. They considered some subclass of a class of all $\zeta^{(s)}$-QSO corresponding to partition $\zeta_{2}$ by choosing coefficients $\left\{P_{i j, k}\right\}_{i j, k=1}^{3}$ in special forms where $a \in[0,1]$, finally they obtained 36 parametric operators. In this paper we are going to study the dynamics of one of these operators which is defined by

$$
v_{a}=\left\{\begin{array}{l}
x^{\prime}=y^{2}+2 y z \\
y^{\prime}=x^{2}+2 a x(1-x) \\
z^{\prime}=z^{2}+2(1-a) x(1-x)
\end{array}\right.
$$

Define $e_{1}, e_{2}, e_{3}$ to be the vertices of the simplex $S^{2}$.

\section{Dynamics of some operators}

Theorem 3.1. Let $\mathrm{V}_{\frac{1}{2}}: \mathrm{S}^{2} \rightarrow \mathrm{S}^{2}$ be a $\zeta^{(\mathrm{s})}-Q S O$ given by (2.3) with $\mathrm{a}=\frac{1}{2}$, then $\operatorname{Fix}\left(\mathrm{V}_{\frac{1}{2}}\right)=\left\{(0,0,1),\left(\frac{1}{3}, \frac{1}{3}, \frac{1}{3}\right)\right\}$.

Proof. In order to find the fixed points we are going to solve the system $V_{\frac{1}{2}}(\mathbf{x})=\mathbf{x}$, where $\mathbf{x}=(x, y, z)$, in other word solving the system

$$
\left\{\begin{array}{l}
x=y^{2}+2 y z \\
y=x^{2}+x(1-x) \\
z=z^{2}+x(1-x)
\end{array}\right.
$$

Subtracting the first and the second equations we get that $(1-2 a) x^{2}+(2 a+2 y+1) x+\left(-3 y+y^{2}\right)=0$. Simplifying this equation we get that $x^{2}+2 a x-2 a x^{2}=y$ substitute $a=\frac{1}{2}$ we get $x^{2}+x-x^{2}=y$, which implies that $x=y$, and implies that $z=1-2 x$. Now substitute $x=y$ and $z=1-2 x$ in the second equation we get that $x^{2}+2 x(1-2 x)=x$ solving this equation we find that $x=0$ and $x=\frac{1}{3}$ are the solutions for the final equation which implies that the points $(0,0,1)$ and $\left(\frac{1}{3}, \frac{1}{3}, \frac{1}{3}\right)$ are two fixed points.

Theorem 3.2. Let $\mathrm{V}_{\mathrm{a}}: \mathrm{S}^{2} \rightarrow \mathrm{S}^{2}$ be a $\zeta^{(\mathrm{s})}-Q S O$ given by (2.3), let $\mathrm{x}_{0}$ be an initial point then

(a) if $\mathrm{x}_{0} \in\left(\frac{1}{3}, 1\right)$, then $\mathrm{V}\left(\mathrm{x}_{0}\right) \in\left(0, \frac{1}{3}\right)$;

(b) the interval $\left[0, \frac{1}{3}\right]$ is an invariant interval;

(c) if $\mathrm{x}_{0} \in\left(0, \frac{1}{3}\right)$, then $\mathrm{V}^{\mathrm{n}}\left(\mathrm{x}_{0}\right) \rightarrow \frac{1}{3}$.

Proof.

(a) Let $x_{0} \in\left(\frac{1}{3}, 1\right)$, so $x_{0}>\frac{1}{3}$, Now since $f(x)=-3 x^{2}+2 x$ is decreasing on $\left(\frac{1}{3}, 1\right)$ we can see that $f\left(x_{0}\right)<f\left(\frac{1}{3}\right)=\frac{1}{3}$ which means that any point in the interval $\left(\frac{1}{3}, 1\right)$ goes to $\left(0, \frac{1}{3}\right)$.

(b) On $\left[0, \frac{1}{3}\right]$ one can see that $V\left(\left[0, \frac{1}{3}\right]\right) \subset\left[0, \frac{1}{3}\right]$ since $f(x)$ is increasing on $\left[0, \frac{1}{3}\right]$ so if $x_{1}<x_{2}<\frac{1}{3}$, then $f\left(x_{1}\right)<f\left(x_{2}\right)<f\left(\frac{1}{3}\right)$.

(c) Since $f(x)>x$ in $\left(0, \frac{1}{3}\right)$ and $f(x)$ is increasing in the same interval $f(f(x))>f(x)$, repeated the same process $n$ times we get that $f^{\mathfrak{n}}\left(x_{0}\right) \rightarrow \frac{1}{3}$. This ends the proof of our theorem. 
Now we consider solving the system $\mathrm{V}^{2}(\mathbf{x})=\mathbf{x}$ in order to find the periodic points of $\mathrm{V}$, where

$$
v_{a}^{2}=\left\{\begin{array}{l}
x^{\prime \prime}=y^{\prime 2}+2 y^{\prime} z^{\prime} \\
y^{\prime \prime}=x^{\prime 2}+2 a x^{\prime}\left(1-x^{\prime}\right) \\
z^{\prime \prime}=z^{\prime 2}+2(1-a) x^{\prime}\left(1-x^{\prime}\right)
\end{array}\right.
$$

Simplifying the system we get that

$$
v_{a}^{2}=\left\{\begin{array}{l}
x^{\prime \prime}=\left(x^{2}+2 a x(1-x)\right)^{2}+2\left(x^{2}+2 a x(1-x)\right) z^{\prime} \\
y^{\prime \prime}=\left(y^{2}+2 y z\right)^{2}+2 a\left(y^{2}+2 y z\right)\left(1-y^{2}-2 y z\right) \\
z^{\prime \prime}=\left(z^{2}+2(1-a) x(1-x)\right)^{2}+2(1-a)\left(y^{2}+2 y z\right)\left(1-y^{2}-2 y z\right)
\end{array}\right.
$$

Theorem 3.3. Let $\mathrm{V}_{\mathrm{a}}: \mathrm{S}^{2} \rightarrow \mathrm{S}^{2}$ be a $\zeta^{(\mathrm{s})}$-QSO given by (2.3), then $\operatorname{per}\left(\mathrm{V}_{\mathrm{a}}\right)=\left\{(0,0,1),(0,1,0),\left(\chi^{*}, 0,1-x^{*}\right)\right\}$ for $0 \leqslant \mathrm{a}<\frac{1}{2}$, and for $\mathrm{a}=\frac{1}{2}$, the points $\left\{(1,0,0),\left(0, \mathrm{y}^{*}, 1-\mathrm{y}^{*}\right)\right\}$ are the periodic points.

Proof. Let us consider two different cases (a) $x=0$; and (b) $x \neq 0$. If $x=0$, then $y+z=1$, now solving the equation $y^{\prime \prime}=y$ we get that $y(2-y)\left(\left(2 y-y^{2}\right)+2 a\left(1-\left(2 y-y^{2}\right)\right)\right)=y$, if $y=0$, then $(0,0,1)$ is a periodic point. But if $y \neq 0$ we get

$$
(2-y)\left(\left(2 y-y^{2}\right)+2 a\left(1-\left(2 y-y^{2}\right)\right)\right)=1, \quad(2 a-1)(y-1)^{2}(2-y)=0 .
$$

Solving the equation we find that either $y=1$, or $y=2$ or $a=\frac{1}{2}$, now if $y=1$, then $z=0$ and so the point $(0,1,0)$ is a periodic point. If $y=2$ it is an impossible case. If $a=\frac{1}{2}$ again we get that $y=y^{*}$ and $z=1-y^{*}$ and so the point $\left(0, y^{*}, 1-y^{*}\right)$ is a periodic point for an arbitrary $y^{*}$ that lies between 0 and 1 .

If $x \neq 0$ we have two different cases either $y=0$, or $y \neq 0$. If $y=0, x+z=1$ now we are going to solve the equation

$$
x=\left(x^{2}+2 a x(1-x)\right)^{2}+2\left(x^{2}+2 a x(1-x)\right)\left((1-x)^{2}+2(1-a) x(1-x)\right),
$$

simplifying the equation we get that

$$
x^{3}+2 x(1-x)+2 a x^{2}(1-x)+4 a(1-x)^{2}=1, \quad(1-x)\left(x^{2}(-1+2 a)+x(1-4 a)+(4 a-1)\right)=0 .
$$

Hence, either $x=1$, and so the point $(1,0,0)$, for $a=\frac{1}{2}$ is a periodic point, or

$$
x^{*}=\frac{-(1-4 a) \pm \sqrt{(1-4 a)^{2}-4(-1+2 a)(4 a-1)}}{2(-1+2 a)} \text { for } a \neq \frac{1}{2} .
$$

So the point $\left(x^{*}, 0,1-x^{*}\right)$ is a periodic point.

Finally we have the case $x \neq 0, y \neq 0$ either $z=0$, or $z \neq 0$. If $z \neq 0$ solving the equation $y^{\prime \prime}=y$ gives us $\left(y^{2}+2 y z\right)^{2}+2 a\left(y^{2}+2 y z\right)\left(1-y^{2}-2 y z\right)=y$, simplifying the equation we get

$$
(1-2 a)(y+2 z)\left(y^{2}+2 y z\right)+2 a(y+2 z)-1=0 .
$$

At $a=\frac{1}{2}$ we get $y+2 z-1=0$, hence $y=1-2 z$ but we know that $x+y+z=1$ substituting the value of $y$ we get that $x=z-1$ which is impossible unless $z=1$, and so $x=0$, and $y=0$ that contradicted our assumption.

Now we are going to divide our simplex $\mathrm{S}^{2}$ into different regions as follows

$$
\begin{array}{rlrlrl}
B_{1} & =\{(x, y, z): x>y>z\}, & B_{2} & =\{(x, y, z): x>z>y\}, & B_{3}=\{(x, y, z): y>x>z\}, \\
B_{4} & =\{(x, y, z): y>z>x\}, & B_{5}=\{(x, y, z): z>x>y\}, & B_{6}=\{(x, y, z): z>y>x\}, \\
\Gamma_{x=y} & =\{(x, y, z): x=y\}, & \Gamma_{x=z} & =\{(x, y, z): x=z\}, & \Gamma_{y=z}=\{(x, y, z): y=z\} .
\end{array}
$$

In the Figure 1 one can see the behavior of the points $(x, y, z)$ in each region.

Depending on the Figure 1 one can see that none of these regions is an invariant region for example for $\mathbf{x} \in \mathrm{B}_{1}$ some points of $V_{a}^{n} \rightarrow(1,0,0)$ and the other goes to $(0,1,0)$. Similarly for the other regions some of their points goes to $(1,0,0)$ and the other goes to $(0,1,0)$. As a special case we can discuss the following case. 


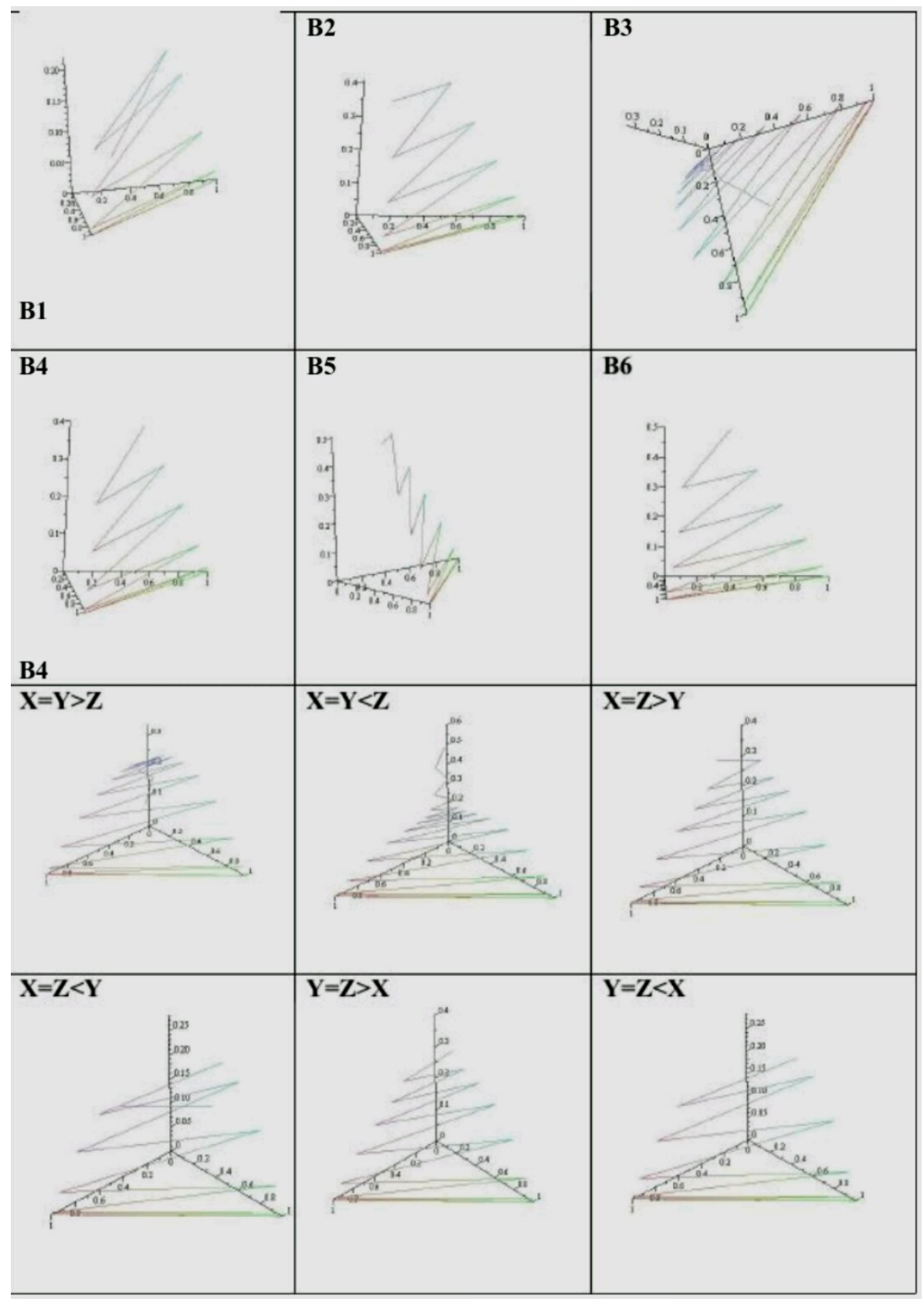

Figure 1: Simplex regions.

Theorem 3.4. Let $\mathrm{V}_{\mathrm{a}}: \mathrm{S}^{2} \rightarrow S^{2}$ be a $\zeta^{(\mathrm{s})}$-QSO given by (2.3), then the line $\mathrm{L}_{\mathrm{y}=0}=\{(x, y, z): y=0\}$ is an invariant line.

Proof. Let $(x, 0, z)$ be any point in $\mathrm{L}_{y=0}$, substitute $y=0$ in (2.3) we get that $x^{\prime}=0, y^{\prime}=x^{2}+2 a x(1-x)$, and $z^{\prime}=z^{2}+2(1-a) x(1-x)$. Now substituting the point $\left(x^{\prime}, y^{\prime}, z^{\prime}\right)$ in the same system we get that $x^{\prime \prime}=y^{\prime 2}+2 y^{\prime} z^{\prime}, y^{\prime \prime}=x^{\prime 2}=0$, and $z^{\prime \prime}=z^{\prime 2}$, doing the same system $n$ times we get that

$$
x^{(n)}=\left(y^{(n-1)}\right)^{2}+2 y^{(n-1)} z^{(n-1)}, \quad y^{(n)}=\left(x^{(n-1)}\right)^{2}, \quad z^{(n)}=\left(z^{(n-1)}\right)^{2},
$$

letting $n$ to infinity we find that $y^{(n)} \rightarrow 0$. And so the line $L_{y=0}$ is an invariant line. 


\section{References}

[1] S. Bernstein, Solution of a mathematical problem connected with the theory of heredity, Ann. Math. Statistics, 13 (1942), 53-61. 1

[2] R. N. Ganikhodzhaev, On a family of quadratic stochastic operators acting in S$^{2}$, Dokl. Akad. Nauk UzSSR., 1 (1989), 3-5. 2

[3] R. N. Ganikhodzhaev, Quadratic stochastic operators, Lyapunov functions, and tournaments, Russian Acad. Sci. Sb. Math., 76 (1993), 489-506. 2, 2

[4] R. N. Ganikhodzhaev, Map of fixed points and Lyapunov functions for a class of discrete dynamical systems, Math. Notes, 56 (1994), 1125-1131.

[5] R. N. Ganikhodzhaev, D. B. Éshmamatova, Quadratic automorphisms of a simplex and the asymptotic behavior of their trajectories (Russian), Vladikavkaz. Mat. Zh., 8 (2006), 12-28. 2

[6] J. Hofbauer, K. Sigmund, The Theory of Evolution and Dynamical Systems: Mathematical Aspects of Selection, Cambridge University Press, Cambridge, (1988). 1

[7] J. Hofbauer, V. Hutson, W. Jansen, Coexistence for systems governed by difference equations of Lotka-Volterra type, J. Math. Biol., 25 (1987), 553-570.

[8] S.-T. Li, D.-M. Li, G.-K. Qu, On stability and chaos of discrete population model for a singlespecies with harvesting, J. Harbin Uni. Sci. Tech., 6 (2006), 10 pages.

[9] A. J. Lotka, Undamped oscillations derived from the law of mass action, J. Am. Chem. Soc., 42 (1920), 1595-1599.

[10] Y. I. Lyubich, Mathematical Structures in Population Genetics, Springer-Verlag, Berlin, (1992). 1

[11] F. Mukhamedov, I. Qaralleh, W. N. F. A. W. Rozali, On $\xi(a)$-quadratic stochastic operators on 2D simplex, Sains Malaysiana, 43 (2014), 1275-1281. 2

[12] F. Mukhamedov, M. Saburov, I. Qaralleh, Classification of $\xi^{(s)}$-Quadratic Stochastic Operators on $2 D$ simplex, J. Phys. Conf. Ser., 2013 (2013), 9 pages.

[13] F. Mukhamedov, M. Saburov, I. Qaralleh, On $\xi^{(s)}$-quadratic stochastic operators on two dimensional simplex and their behavior, Abstr. Appl. Anal., 2013 (2013), 12 pages. 1, 2

[14] M. Plank, Hamiltonian structures for the n-dimensional Lotka-Volterra equations, J. Math. Phys., 36 (1995), $3520-3534$. 1

[15] F. E. Udwadia, N. Raju, Some global properties of a pair of coupled maps: quasi-symmetry, periodicity, and synchronicity, Phys. D, 111 (1998), 16-26. 1

[16] V. Volterra, Lois de fluctuation de la population de plusieurs espéces coexistant dans le méme milieu, Association Franaise pour l'Avancement des Sciences, Lyon, (1926). 1 